\title{
Continuum Model Analysis of QCM Nanotribological Data to Obtain Friction Coefficients for 304SS Contacts Lubricated by Water and $\mathrm{TiO}_{2}$ Nanoparticle Suspensions
}

\author{
Caitlin M. Seed, Biplav Acharya and Jacqueline Krim* \\ Department of Physics, North Carolina State University, Raleigh, NC, United States
}

\section{OPEN ACCESS}

Edited by:

Valentin L. Popov,

Technical University of

Berlin, Germany

Reviewed by:

Guoxin Xie,

Tsinghua University, China

Qunyang Li,

Tsinghua University, China Motohisa Hirano,

Hosei University, Japan

*Correspondence: Jacqueline Krim jkrim@ncsu.edu

Specialty section:

This article was submitted to

Tribology,

a section of the journal

Frontiers in Mechanical Engineering

Received: 01 June 2020

Accepted: 27 July 2020 Published: 08 September 2020

Citation:

Seed CM, Acharya B and Krim J (2020) Continuum Model Analysis of QCM Nanotribological Data to Obtain Friction Coefficients for 304SS

Contacts Lubricated by Water and $\mathrm{TiO}_{2}$ Nanoparticle Suspensions.

Front. Mech. Eng. 6:72.

doi: 10.3389/fmech.2020.00072
We report a study of the response of a Quartz Crystal Microbalance (QCM) to rubbing contacts in air, water and aqueous suspensions of $40 \mathrm{~nm} \mathrm{TiO}_{2}$ nanoparticles. Measurements were performed with a contact comprised of 3 close-packed 304SS ball bearings situated symmetrically about the center of a 304SS QCM electrode with $2 \mathrm{~nm}$ rms roughness. Two continuum methods were employed to infer macroscale friction coefficients $\mu$ employing QCM nanotribological data recorded in the Cattaneo-Mindlin (CM) slip regime at vibrational amplitudes that varied between 1 and $17 \mathrm{~nm}$. The "slope" Method 1 involved sweeps of the QCM amplitude of vibration as ball bearings were held in continuous contact with the oscillating electrode. The "contact" Method 2 obtained $\mu$ by analyzing the shifts in frequency and bandwidth that occur at a fixed $u_{0}$ to solve for $\mu$. when ball bearings were brought in and out of contact with the QCM's electrode. The results for dry and water lubricated contacts compared favorably with macroscale friction coefficients reported in the literature. The model failed to adequately describe contacts lubricated with the NP suspension, but its continuum nature did not appear to be the dominant factor underlying failure. The failure was more likely attributable to either a lack of a CM slip regime when NP were present at the interface and/or the fact that the amplitude of vibration was close in size to the individual NP contacting regions, in violation of a key underlying assumption of the model.

Keywords: friction, QCM, cattaneo-mindlin, nanotribology, contact mechanics

\section{INTRODUCTION}

Quartz Crystal Microbalance (QCM) studies of tribological contacts (Rodahl and Kasemo, 1996; Laschitsch and Johannsmann, 1999; Brizmer et al., 2007; Johannsmann, 2007; Dawson et al., 2009; Krim, 2012; M'boungui et al., 2014; Borovsky et al., 2017) at both the macro and nanoscale are of increasing importance in a wide range of nano-technological and energy-related applications (Braiman et al., 2003; Kim and Kim, 2009; Zhang and Li, 2010; Krim, 2012; Hsu et al., 2014). Literature reports in this area have, to date, focused primarily on either macroscale or nanoscale contacts, and a remaining challenge in the field of tribology is to establish linkage between studies performed at wide-ranging length and time scales. One approach to establishing this linkage is to perform studies that examine the limits of macroscopic continuum mechanics, by probing where 
continuum methods break down as the atomic scale is approached. Nanoparticle (NP) additives are attractive candidates for such studies, as they significantly alter friction and wear at macroscopic length scales when present in a lubricated contact (Yu et al., 2016).

We report here a study of the response of QCMs with 304 Stainless Steel (304SS) sensing electrodes to rubbing contact with 304SS ball bearings, employing a continuum model to analyze the response in air, water and an aqueous $\mathrm{TiO}_{2}$ suspensions. The QCM technique yields real-time nanotribological information at macroscale contact velocity amplitudes, typically $0.1-1 \mathrm{~m} / \mathrm{s}$. It is comprised of a thin piezoelectric quartz crystal driven at its resonance frequency by applying an AC voltage to thin metal film electrodes deposited onto each of its major faces. Contact forces and/or changes in the environment surrounding the QCM's surface electrode produce shifts in both its resonance frequency $f$, and quality factor $Q$, and the response of these quantities to probes, biological agents, and/or granular materials has been extensively studied (Borovsky et al., 2001, 2007; Krim, 2012; Dultsev and Nekrasov, 2018; Dirri et al., 2019; Han et al., 2019; Wang et al., 2019). Analysis approaches for inferring macroscopic friction coefficients from such measurements (Berg and Johannsmann, 2003; Berg et al., 2003; Leopoldes and Jia, 2010; Hanke et al., 2013; Vlachová et al., 2015; Borovsky et al., $2017,2019)$ have been largely developed within the context of continuum contact models. The models must eventually break down as nanoscale length scales are approached, where systems become less uniform in nature and/or as contact regions become more poorly defined (Figure 1).

Vlachová et al. (2015) recently utilized a Cattaneo-Mindlin (CM) slip scenario to relate contact stiffness obtained from QCM measurements to macroscale friction coefficients, employing an analysis approach developed by Johannsmann and coworkers for QCM's loaded with spherical contacts (Berg and Johannsmann, 2003; Berg et al., 2003; Hanke et al., 2013). The CM scenario is an approximation that allows for a simplified analysis framework for two curved surfaces that are pressed with a normal load $F_{N}$ into contact and then sheared tangentially (Etsion, 2010; Paggi et al., 2014; Ciavarella, 2015). Such bodies commonly remain stuck to each other in one part of the contact while slipping in other regions. The CM approximation treats the contact as rigid indenter pressed into a flat elastic surface, and assumes that sliding friction in the slip region is governed by a "Coulomb" friction law, $F_{f}=\mu_{k} F_{N}$.

Berg and Johannsmann (2003), Berg et al. (2003), Hanke et al. (2013), and Vlachová et al. (2015) analysis method is based on both the linear and non-linear contact mechanics regimes associated with partially slipping contacts undergoing small amplitude reciprocal motion. It assumes that the contact area diameter is both independent of the tangential loading force and much smaller than the loading sphere diameter. Vlachová et al. obtained realistic values for friction coefficients $\mu_{k}$ by analyzing QCM frequency and bandwidth shifts, $\Delta f$ and $\Delta \Gamma=(f / 2) \Delta\left(Q^{-1}\right)$, as a function of the amplitude of

Abbreviations: QCN, Quartz Crystal Microbalance; CM, Cattaneo-Mindlin; SS, Stainless Steel; BB, Ball Bearing. vibration for glass spheres in contact with silica or polymercoated QCM electrodes. The values were not, however, compared to conventional macroscopic measurements of $\mu_{k}$ on the same materials combinations. Seed et al. (2020) very recently employed the Vlachová et al. (2015), Berg and Johannsmann (2003), Berg et al. (2003), and Hanke et al. (2013) analysis approach to infer macroscale friction coefficients $\mu$ for QCMs loaded with stainless steel spherical contacts, and also measured $\mu$ in conventional macroscale setups for the same materials combinations. They found that the values agreed well when the dependence of $\Delta \Gamma$ on vibrational amplitude was utilized. Overall, Seed et al. validated the combined assumptions of the continuum analysis methods, and demonstrated that the Vlachová et al. (2015), Berg and Johannsmann (2003), Berg et al. (2003), and Hanke et al. (2013) approach was viable for linkage macro and nanoscale tribological measurements (Seed et al., 2020).

The study reported by Seed et al. was performed in air on unlubricated stainless steel contacts, and the analysis was performed from a continuum perspective (Seed et al., 2020). We employ it here to examine both dry and lubricated contacts, with a particular focus on the degree to which such a continuum model can be applied to a complex liquid-nanoparticle-solid interface. $\mathrm{TiO}_{2}$ suspensions were selected for study, as they have been reported on extensively in the tribological literature and are known to reduce friction in stainless steel contacts at both the nanoscale and macroscale (Glavatskih and Höglund, 2008; Ali et al., 2016; Acharya et al., 2019a; Wu et al., 2020). $\mathrm{TiO}_{2}$ suspensions are also of interest in tribotronics ["active" or "smart" control of friction by combining mechanical elements with electronics (Glavatskih and Höglund, 2008; Zhang and Wang, 2016; Krim, 2019)], since they are readily repositioned by external fields for purposed of tuning of friction levels (Acharya et al., 2019b; Krim, 2019). In addition, the suspensions are of interest in energy storage applications utilizing flow batteries, where interfacial tribological properties are of importance (Wang et al., 2007; Sen et al., 2017).

\section{MATERIALS AND METHODS}

Measurements were performed in air, deionized (DI) water, and DI water with suspensions of $0.67 \mathrm{wt} \% \mathrm{TiO}_{2}$ nanoparticles in an apparatus depicted schematically in Figure 2. The apparatus was comprised of a Ball Bearing (BB) configuration with three 304SS bearings arranged in a close packed triangular array centered on the QCM upper (liquid facing) electrode. The BB's made contact with the upper electrode at a distance of $2.29 \mathrm{~mm}$ from the center, which fell within the perimeter of the lower electrode and therefore within an area of active oscillation. The system weighed $F_{N}=0.282 \mathrm{~N}$, distributed across the three contacts so as to load $0.094 \mathrm{~N}$ onto each of them. This loading configuration has a Hertzian contact pressure of $245 \mathrm{MPa}$ in air and a contact radius of $11.05 \mu \mathrm{m}$ under the assumption of a circular contact (Popov, 2010). Measurements were performed in reciprocal motion with maximum sliding speed amplitudes ranging between 0.03 and $0.22 \mathrm{~m} / \mathrm{s}$ (liquid) or $0.53 \mathrm{~m} / \mathrm{s}$ (air) at the point of contact. Under these conditions, the system falls within 


\section{Nanoscale perspective}

\section{Continuum perspective}

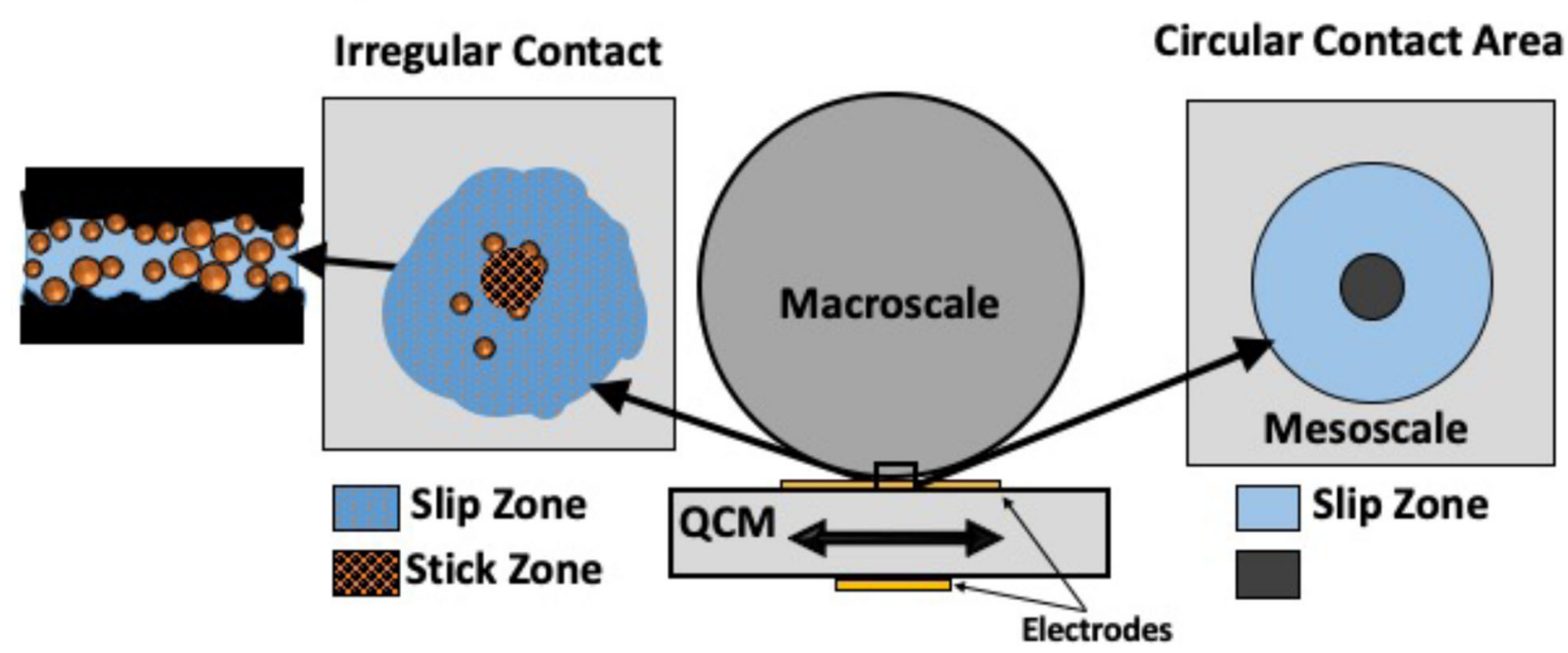

FIGURE 1 | Nanoscale and Continuum perspectives of one ball bearing on an oscillating QCM displaying Cattaneo-Mindlin slip, with the contact area having an inner stick zone and an outer slip zone.

the boundary lubrication regime where direct contact between asperities supports the load ( $\mathrm{Lu}$ et al., 2006; Yamaguchi and Hokkirigawa, 2016). The liquid nonetheless penetrates the gaps in contact associated with roughness, allowing introduction of NP. For the liquid measurements, $0.6 \mathrm{~mL}$ of either DI water or the $\mathrm{TiO}_{2}$ suspension was added to evenly cover the QCM, resulting in the tripod BBs being slightly more than half submerged. The associated buoyant force of $0.00086 \mathrm{~N}$ was significantly smaller than the normal force, but was nonetheless included in the model calculations. The system components were rigidly attached to each other, and, in contrast to the system employed by Vlachová et al. were close packed. Differences in the results associated with the different experiment geometries are however expected to be minimal, as the tangential forces and local contact geometries were very similar.

\section{Materials}

Anatase $\mathrm{TiO}_{2}$ (titania) nanoparticles with density, Zeta potential, and radius, respectively, $=4.23 \mathrm{~g} / \mathrm{cm}^{3},-32.7 \mathrm{mV}$ and $20 \mathrm{~nm}$ were obtained from US Research Nanomaterials (stock number: US7071 Houston, TX 77084, USA) at concentration $\sim 20$ wt $\%$ and were diluted to a concentration of $0.67 \mathrm{wt}$. The $\mathrm{NP}$ suspension, as prepared, had a $\mathrm{pH}$ of 7.7 and density of $1.00367 \mathrm{~g} / \mathrm{cm}^{3}$. NP aggregation was not observed, but some sedimentation was observed for samples left unstirred for periods of $12-24 \mathrm{~h}$. The sedimentation was readily reversible by stirring and sonication and the suspensions were stirred and sonicated for $10-15 \mathrm{~min}$ immediately before each experimental run, with measurements being completed within $60 \mathrm{~min}$ thereafter. All data were recorded within $48 \mathrm{~h}$ of initial preparation of the suspension.
Bulk 0.2 g 5/32" diameter Grade 100 (G100) 304SS bearings with maximum rms surface roughness $5.0 \mu$ in $(127 \mathrm{~nm})$ were attached to the underside of a slider, as depicted in Figure 2. They were glued onto the holder using Gorilla Super Glue (Gorilla Glue Company, Sharonville, OH, USA).

The QCM crystals employed for the studies were AT-cut, 5 $\mathrm{MHz}$, one-inch diameter QCM crystals with 304SS electrodes (FILTECH, Inc. part no. QM1022, Boston, MA). The crystals had half-inch diameter surface electrodes made of 304SS and quarter inch backside electrodes made of Au. The 304SS electrodes consisted of $200 \mathrm{~nm} 304$ SS films deposited atop $100 \mathrm{~nm}$ thick $\mathrm{Au}$ films deposited on $50 \mathrm{~nm}$ thick Ni adhesion layers, with rms surface roughness $2 \pm 1 \mathrm{~nm}$ (Acharya et al., 2018).

\section{Methods \\ Contact Angle}

Contact angle measurements were performed as an independent indication of NP suspension potential for lubricity. The measurements were performed on the actual samples used for the measurements, as contact angles, and also lubricating properties, are highly dependent on surface roughness, NP concentrations, NP size and interfacial chemical compositions (Wu et al., 2020). Contact angles were measured for both DI water and $\mathrm{TiO}_{2}$ suspensions by using a pipette to place a $10 \mu \mathrm{L}$ drop onto the 304SS QCM electrode. An image was used to determine the contact angle on both sides of the droplets by drawing a right triangle where the base was the surface from the edge of the droplet to the center, and a line was drawn tangent to the droplet edge, forming the hypotenuse. The value for contact angle displayed in Figure 3 is the average of the right and left angles.

The $\mathrm{TiO}_{2}$ suspension exhibited a higher contact angle than that for pure water, indicating that a significantly larger slip 

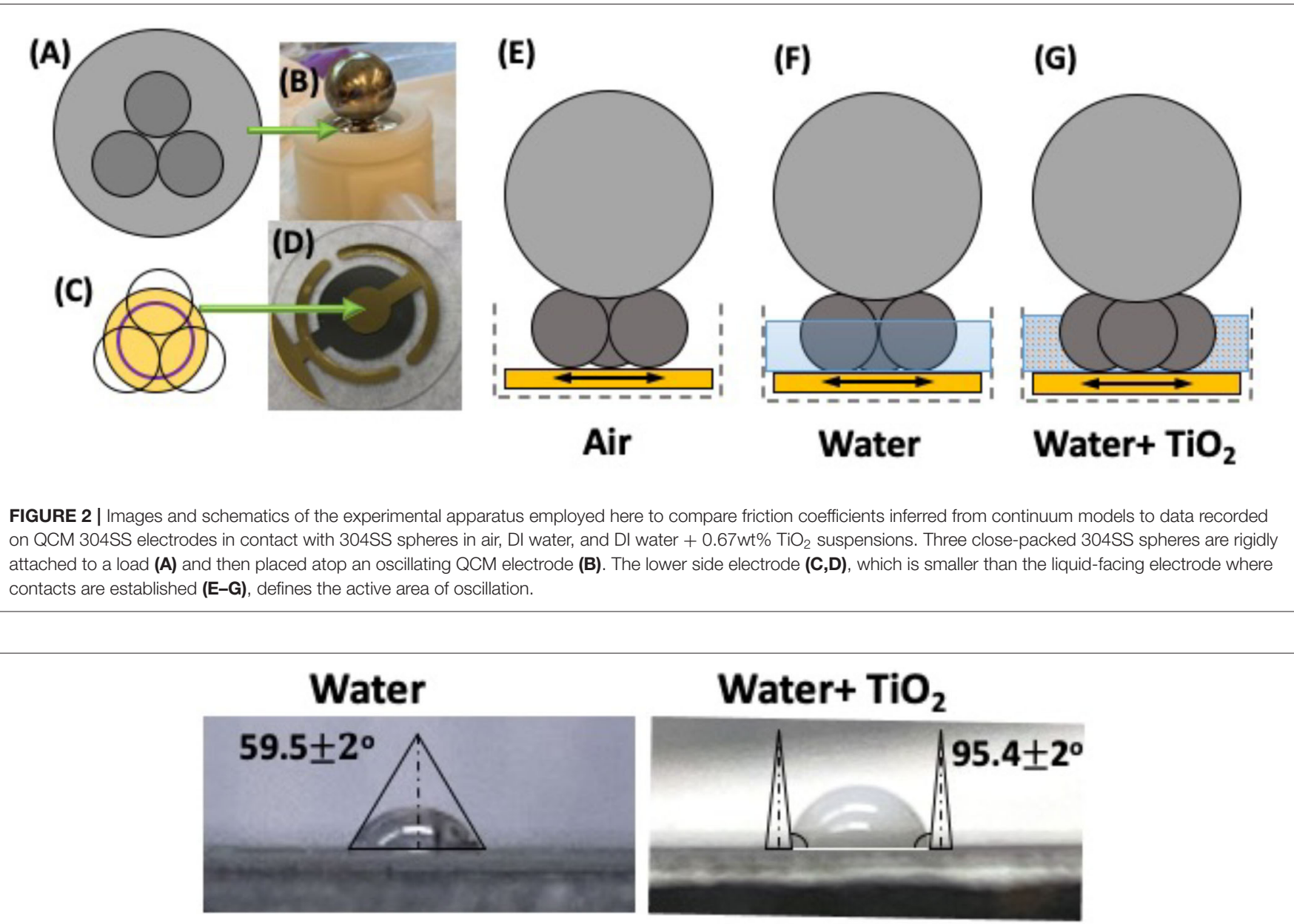

Water+ $\mathrm{TiO}_{2}$

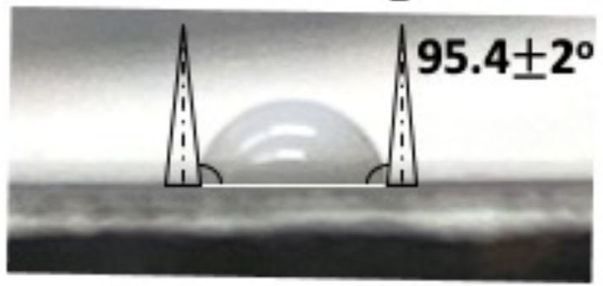

FIGURE 3 | Images of sessile droplets of (left) pure DI water, and (right) a 0.67 wt\% suspension of $\mathrm{TiO}_{2} \mathrm{NP}$ atop the QCM $304 \mathrm{SS}$ electrode, along with droplet tangents drawn to compute the contact angle.

length along with a lower friction coefficient is to be expected for contacts completely immersed in the liquid (Ellis et al., 2003; Acharya et al., 2019b). We note that this is distinct from the wetting and spreading of lubricant droplets, which also play major roles in the lubrication of dynamic mechanical systems (Wu et al., 2020).

\section{QCM Continuum Model Analysis}

Data recorded here were analyzed according to the methods described in Berg and Johannsmann (2003), Berg et al. (2003), Hanke et al. (2013), Vlachová et al. (2015), and Seed et al. (2020), which are applicable to the CM partial slip approximation in a small amplitude reciprocal motion (Figure 1). For reciprocal motion, and as the amplitude of motion increases, the partial slip regime in this model commences at the contact's edge in the form of thin annulus. The annulus progressively grows and the system transitions to full gross slippage for a sufficiently large applied tangential force (Figure 4).

Values for $\mu$ in the slipping region of the contact are inferred from the shifts in resonance frequency, $\Delta f$, and half bandwidth at half height ("bandwidth," for short), $\Delta \Gamma$, of the QCM oscillating at $\mathrm{MHz}$ resonance frequency upon contact of a sphere with the surface (We note here that the quality factor $\mathrm{Q}$, damping parameter $\mathrm{D}_{\mathrm{f}}$, and dissipation $\mathrm{D}$, (all unitless) are all in common use to represent system dissipation. They are related to $\Delta \Gamma$, which has units of $\mathrm{Hz}$, as The model developed by Johannsmann and coworkers treats the system as a composite mechanical resonator consisting of a QCM in contact with a fixed load, with contact comprised of a dashpot in parallel with a spring. On a basic level, $\Delta f$ and $\Delta \Gamma$ are proportional to the in-phase and out-of-phase component of the area-averaged periodic tangential stress at the resonator surface (Berg et al., 2003), and can be inferred from expressions for the tangential force and contact area. The time dependent tangential force at the contact is written as Hanke et al. (2013):

$$
F_{x}(t)=\kappa u(t)+\xi v(t)
$$

where $\mathrm{u}$ is the tangential displacement, $\kappa$ is the contact stiffness, $\mathrm{v}$ is the tangential sliding speed and $\xi$ is a linear drag coefficient. For a viscoelastic contact small amplitude cyclic motion with 


\section{(A) Non-slipping contact, (B) Cattaneo-Mindlin (C) Fully sliding contact, viscoelastic dissipation partial slip scenario velocity independent friction}

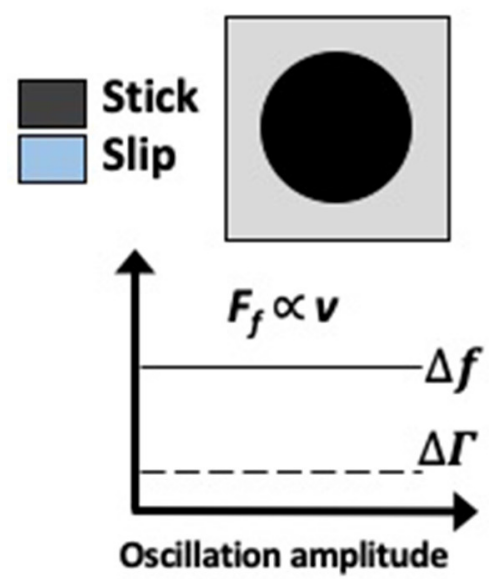

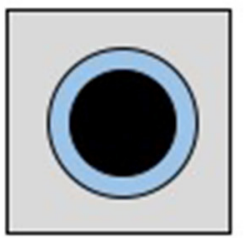

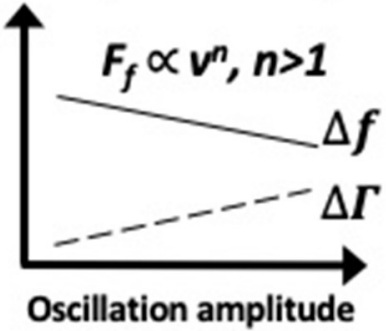

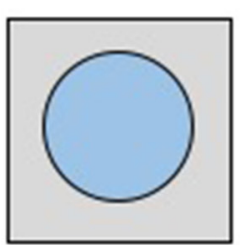

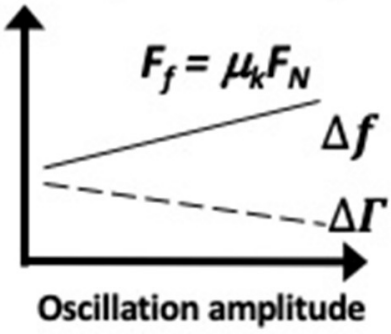

FIGURE 4 | Schematic of the $\Delta f$ and $\Delta \Gamma$ response of a QCM in three distinct idealized continuum contact conditions (A) A non-slipping viscoelastic contact, (B) A partially slipping contact with slipping regions governed by Coulomb friction, and (C) a fully slipping contact governed by Coulomb friction.

amplitude $u_{o}$ and tangential velocity amplitude $v_{o}=i \omega u_{o}$, the contact area $A_{\text {eff }}$ is constant, all forces depend linearly on displacement, and the complex frequency shift associated with the presence of the contact can be expressed as Laschitsch and Johannsmann (1999) and Hanke et al. (2013):

$$
\Delta f+i \Delta \Gamma=\frac{n_{p} \kappa}{2 n \pi^{2} A_{e f f} Z_{q}}(\kappa+i \omega \xi),
$$

where $A_{\text {eff }}$ is the effective area of the resonator, $\kappa$ is the contact stiffness, $n$ is the overtone order, $F_{N}$ is the load, and $Z_{q}=8.8 * 10^{6}$ $\mathrm{kg} /\left(\mathrm{m}^{2} \mathrm{~s}\right)$ is the shear wave impedance. The quantities in Equation (2) are amplitude independent, and therefore both $\Delta f$ and $\Delta \Gamma$ are constant in amplitude (Figure 4A). For the case of full slip governed by a velocity independent Coulomb friction law, $\Delta f$ trending linearity upward and $\Delta \Gamma$ trends linearly downward as the amplitude increases (Figure 4C) (Vittorias et al., 2010).

For the case of a composite contact that is partially slipping, $\mathrm{F}_{\mathrm{X}}(\mathrm{t})$ is not time harmonic, and the analysis must encompass both linear and non-linear contact mechanics regimes with proper matching of boundary conditions. The analysis developed in Berg and Johannsmann (2003), Berg et al. (2003), Hanke et al. (2013), Vlachová et al. (2015) reveals that in this regime $\Delta f$ trends downward with amplitude while $\Delta \Gamma$ trends upward (Figure 4B). To emphasize the linear trends, the expressions in Vlachová et al. (2015), Equation 11 can be rewritten as Seed et al. (2020):

$$
\begin{aligned}
& \Delta f\left(u_{o}\right)=h-b u_{o} \\
& \Delta \Gamma\left(u_{o}\right)=\frac{4}{3 \pi} b u_{o} \\
& h=\frac{n_{p} \kappa}{2 n \pi^{2} A_{\text {eff }} Z_{q}}
\end{aligned}
$$

$$
b=\frac{n_{p} \kappa^{2}}{6 n \pi^{2} A_{e f f} Z_{q} F_{N}} \frac{1}{\mu}
$$

Where $n_{p}$ is the number of BB contacts, $\mu$ is the coefficient of friction, and $u_{o}$ is the amplitude of oscillation at the center of the QCM (Vlachová et al., 2015). The latter quantity is inferred from Hanke et al. (2013):

$$
u_{0}=\frac{\alpha d_{q}}{2 \omega e_{26} A_{e f f}} i
$$

where the parameter $\alpha$ is a numerical factor set equal to 1 since the QCM is modeled as a piezoelectric parallel plate, $d_{q}$ is the thickness of the QCM, $e_{26}=9.65 \times 10^{-2} \mathrm{C} / \mathrm{m}^{2}$ is the piezoelectric stress coefficient, $i$. is the current, and $\omega$ is the angular resonant frequency of the QCM (Hanke et al., 2013). In addition to the above formulation, a heuristic term representing losses linear in stress $\Delta \Gamma_{\text {off }}$ can be added to the term in Equation (4), which offsets the curve by a constant (Vlachová et al., 2015).

The value for the friction coefficient $\mu$ is inferred via two distinct methods, which were referred to by Seed et al. as "Method 1" and "Method 2," referred to herein as "slope Model 1 " and "contact Model 2." The slope Method 1 substitutes the slope of $\Delta \Gamma$ vs. $u_{o}$, as determined from an amplitude sweep measurement, into Equation (4) to solve for $b$. The $y$ intercept of a linear fit to the $\Delta$ f vs. $u_{o}$ data meanwhile yields a value for $h$. The values for $b$ and $h$ are then substituted into Equations $(5,6)$ to obtain $\mu$. The contact Method 2 utilizes the $\Delta \mathrm{f}$ and $\Delta \Gamma$ shifts that occur at a fixed $u_{o}$ to solve for $\mu$.

Both methods must be evaluated in the $\mathrm{CM}$ slip regime, which is a basic assumption of the model, and predicts a negative (positive) linear slope in frequency (bandwidth) with amplitude. Friction coefficients were therefore evaluated only in amplitude 
regions exhibiting these linear trends, and those regions were denoted as CM slip regimes. The value of $h$ was determined from the intercept of the $\Delta \mathrm{f} v$ s. $\mathrm{u}_{\mathrm{o}}$ linear fit in the CM slip regime, while the value for b was obtained from the $\Delta \Gamma$ vs. $u_{o}$ slope in the same regime. Since Equation 4 has a zero intercept, a requirement was in general enforced when finding the $\Delta \Gamma$ data fit, with the slope of this line used as the value of $b$. This condition was relaxed however for fits to the contacts lubricated by the NP suspension, as the nature of the contact geometry might be expected to change for low vibrational amplitudes.

The friction coefficient, which is a kinetic friction coefficient as determined by Seed et al. is obtained by solving Equations (5, 6) for $\mu$ in terms of $h$ and $b$ (Seed et al., 2020):

$$
\mu=\frac{2 \pi^{2} n A_{e f f} Z_{q}}{3 n_{p} F_{N} b} h^{2}
$$

For the present studies, a value of $A_{\text {eff }}=3.165 \times 10^{-5} \mathrm{~m}^{2}$ was employed for the effective area of oscillation, corresponding to the area of the smaller electrode, along with $f_{0}=5 \mathrm{MHz}$ for the fundamental $(n=1)$ resonant frequency, $\mathrm{n}_{\mathrm{p}}=3$ for the number of BBs in contact with the QCM, and the acoustic impedance value $Z_{q}=8.8 \times 10^{6} \mathrm{~kg} /\left(\mathrm{m}^{2} \mathrm{~s}\right)$.

For the contact Method 2, the measured values of $\Delta f$ and $\Delta \Gamma$ at a fixed oscillation amplitude in the CM Slip allow evaluation of $\mu$ by direct substitution of Equation (3) into Equation (4), followed by some rearrangement of terms:

$$
\mu=\frac{4 \beta \kappa^{2}}{9 \pi F_{N} \Delta \Gamma} u_{o}
$$

where $\beta$ and $\kappa$ are given by:

$$
\beta=\frac{n_{p}}{2 n \pi^{2} A_{e f f} Z_{q}}, \quad \kappa=\frac{\Delta f+\frac{3 \pi}{4} \Delta \Gamma}{\beta}
$$

It is noted here that the contact Method 2 is straightforward in that it requires only a single measurement of $\Delta f$ and $\Delta \Gamma$ to obtain a value for $\mu$. The analysis is only valid however if measurement is performed at an amplitude of vibration where CM slip is occurring. This can be confirmed by imaging the contact zone or alternatively performing a local amplitude sweep that confirms a negative (positive) and linear slope for $\Delta f(\Delta \Gamma)$ vs. $u_{o}$ in the region being studied.

\section{Quartz Crystal Microbalance Apparatus and Data Recording}

Measurements were performed with no contacting sphere by first installing the QCM into a Teflon holder and allowing the frequency to stabilize in air or, for the case of liquid measurements, in liquid. The tripod BB contacts were then loaded onto the QCM electrode while the system response was recorded, beginning with the minimum amplitude of vibration, and continuing on as the amplitude of vibration was increased. Variations in amplitude were achieved by introducing electrical resistance, ranging from $1,190 \Omega$ to $0 \Omega$, between the QCM electrode and the oscillator circuit. This procedure was starting with $1,190 \Omega$ and subsequently reduced every $60 \mathrm{~s}$ until a value of $0 \Omega$ was attained. A baseline resonance frequency was established by using the same process for changing the QCM amplitude on the same QCM without the BB load, and for immersed contacts in the case of the lubricated measurements. Plots of $\Delta \mathrm{f}$ and $\Delta \Gamma$ vs. $\mathrm{u}_{\mathrm{o}}$ were then generated. The values from these curves at equal values for $\mathrm{u}_{\mathrm{o}}$ were used to solve for $\mu$ via the contact Method 2 . To take into account the fact that the amplitude of vibration at the contact points was lower in magnitude than at the center of the electrode, the amplitude at the contacts was a modeled as a Gaussian decay, and the central maximum was calculated from Equation 11, according to Martin and Hager (1989), Josse and Lee (1998), and Lu et al. (2004):

$$
u=u_{o} e^{-\frac{a r^{2}}{R_{e}^{2}}}
$$

where $a=2$ is an estimated constant, $r=2.29 \mathrm{~mm}$ is the distance from the center, and $\mathrm{R}_{\mathrm{e}}=3.175 \mathrm{~mm}$ is the radius of the active oscillation region of QCM electrode. Slopes of the plots were then utilized to evaluate $\mu$.

A QCM100 (Stanford Research Systems, Sunnyvale, CA, USA) system with the included oscillator driving circuitry, controller, and Teflon sample holder was employed to record the QCM measurements. A frequency counter (HP 53181A, Keysight Technologies, Santa Rosa, CA) and a multimeter (Keithley 2000 Series, Tektronix, Inc., Beaverton, OR) were used to measure the QCM frequency and conductance voltage via a LabView (National instruments, Austin, TX) data acquisition system. Conductance voltage $V_{c}$ was converted to motional resistance $\mathrm{R}$ according to $\mathrm{R}=10^{\left(4-V_{c} / 5\right)}-75^{1}$, and shifts in $\mathrm{R}$ were converted to shifts in $\Gamma$ according to Johannsmann et al. (2009):

$$
\Delta \Gamma=\frac{16 A_{e f f} f_{0}^{3} Z q d_{26}^{2}}{\pi} \Delta R
$$

where $f_{0}$ is the fundamental resonant frequency ( $\mathrm{n}=1$ for these studies $), Z_{q}=8.8 \times 10^{6} \mathrm{~kg} /\left(\mathrm{m}^{2} \mathrm{~s}\right)$ is the acoustic impedance, and $d_{26}=3.1 \times 10^{-12} \mathrm{~m} / \mathrm{V}$ is the piezoelectric strain coefficient.

\section{RESULTS}

Figure 5 presents amplitude sweep data sets recorded for the response of the QCM in continuous contact with the tripod in the three environments studied. Values for $\mu$ displayed in lower panel of Figure 5 were evaluated in the respective CM regimes using the contact Method 2. All data are plotted vs. the amplitude at the actual contact (i.e., not the amplitude at the center of electrode) and range from 1 to $17 \mathrm{~nm}$ for air and 1 to $7 \mathrm{~nm}$ for the liquids, which, respectively, correspond to velocity amplitudes of $0.03-$ $0.53 \mathrm{~m} / \mathrm{s}$ and $0.03-0.22 \mathrm{~m} / \mathrm{s}$. Notably, the maximum amplitude in all cases was $<40 \mathrm{~nm}$ diameter of the $\mathrm{TiO}_{2} \mathrm{NP},>2 \mathrm{~nm} \mathrm{rms}$ roughness of the $304 \mathrm{SS}$ electrodes but $<127 \mathrm{~nm}$ maximum rms roughness of the 304SS BB's. Regions of the amplitude sweep that are consistent $\mathrm{CM}$ slip are shaded orange and designated

${ }^{1}$ QCM200 Quartz Crystal Microbalance Digital Controller - QCM25 $5 \mathrm{MHz}$ Crystal Oscillator, Revision 2. 


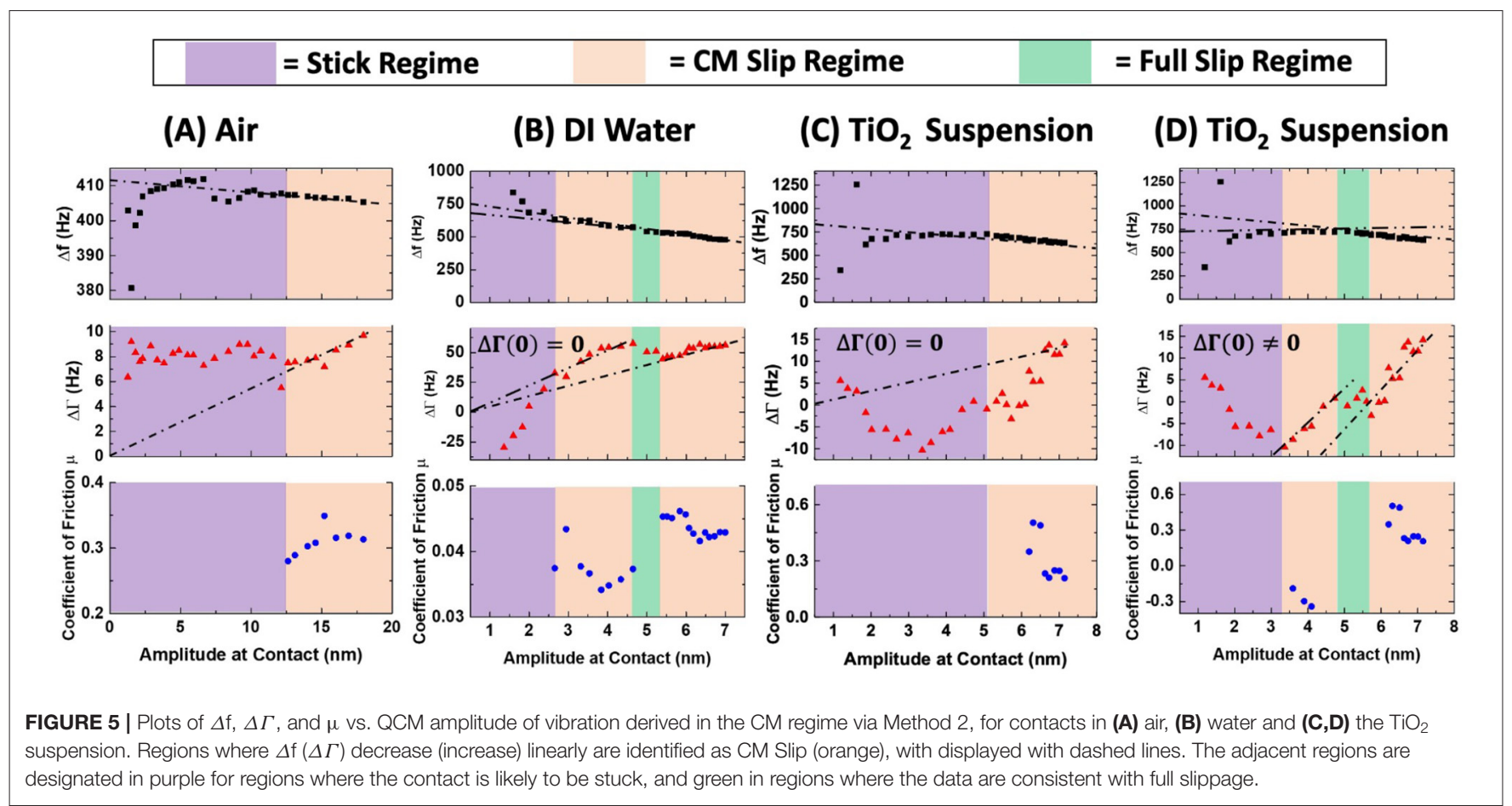

as "CM slip." The amplitude region falling between zero and the first CM slip regime is shaded purple and arbitrarily labeled the "stick regime" since the contacts are likely to transition to being stuck at the lowest amplitudes For the lubricated contacts, there are multiple regions that are consistent with CM slip (e.g., Figure 4B) that are separated by regions consistent with full slip (e.g., Figure 4C). For the water lubricated contacts the $\Delta \Gamma$ vs. amplitude data in the $\mathrm{CM}$ slip regimes trend toward zero at zero amplitude. It is difficult to identify a region of clear CM slip for the NP suspensions, as there is no extended region where the $\Delta \Gamma$ data exhibits a positive linear slope that extrapolates to zero.

Values for friction coefficients obtained inferred from the data displayed in Figure 5 in the CM slip regimes are presented in Table 1, as well as the amplitude of vibration at which the transition from stick to a CM partial slip condition is designated. The slope Method 1 values employed the slope and intercept values from the fits displayed as black dotted lines to the $\Delta \Gamma$ data in Figure 5. The contact Method 2 value are inferred from the values of $\Delta f$ and $\Delta \Gamma$ at fixed amplitudes, as denoted in the table. Since the NP suspension $\Delta \Gamma$ data do not trend toward zero at zero amplitude, the slope Method 1 was also applied by employing fits that were either required or not required to have a zero intercept. Relaxing the zero-intercept condition resulted in a significantly lower value for $\mu$ that was also more realistic, as discussed next.

\section{DISCUSSION}

Prior measurements employing the same experimental configuration have indicated the amplitudes inferred from
Equation 11 may be underestimated, causing the values for friction coefficients reported in Table 1 to be slightly underestimated by $\sim 25 \%$ (Seed et al., 2020). Inspection of the results in Figure $\mathbf{5}$ and Table $\mathbf{1}$ for contacts in air, nonetheless reveals values of $\mu \sim 0.3$, which are in close agreement in close agreement with conventional ball on disk macroscale measurements (Seed et al., 2020). The continuum model therefore successfully treats this system, irrespective of the fact that the amplitude of vibration is clearly nanoscale in extent.

For the case of contacts immersed in water, the Method 1 and 2 values of $\mu \sim 0.04$ in region 1 agree closely, and are close for the second region, at $\mu \sim 0.045 \& 0.064$. The values are also of the same order as conventional ball on disk macroscale measurements for water-immersed 304SS contacts (Curtis et al., 2017), where $\mu \sim 0.1$ but falling slightly lower. Overall, the continuum model can be considered to successfully treat this system, with the QCM data revealing both a lower friction coefficient and amplitude of transition from stick to partial slip that has been reduced to less than half of the value in air.

The analysis methods yield results that are far more problematic for contact immersed in the $\mathrm{TiO}_{2}$ suspension. $\mathrm{TiO}_{2}$ suspensions are known to lower the macroscopic friction coefficient for pure water by as much as $20-50 \%$ (Ali et al., 2016; Pardue et al., 2018; Acharya et al., 2019a; Wu et al., 2020), and this is not reflected in the Table 1 values. The Method 1 and 2 values of $\mu$ are moreover in clear disagreement with each other, and clear regions of CM slip are difficult to define. Realistic values for the friction are obtained by relaxing the zero-intercept condition. Without relaxed conditions, the model clearly fails upon introduction of NPs. The zero-intercept 
TABLE 1 | Friction coefficients inferred from the Figure 5 data by two analysis methods, along with the transition amplitude of vibration from stick to partial slip.

\begin{tabular}{|c|c|c|c|c|}
\hline & Air & DI Water & $\begin{array}{c}0.67 \mathrm{wt} \% \mathrm{TiO}_{2} \\
\Delta \Gamma(\mathrm{u}=0)=0\end{array}$ & $\begin{array}{c}0.67 \text { wt } \% \mathrm{TiO}_{2} \\
\Delta \Gamma(\mathrm{u}=0) \neq 0\end{array}$ \\
\hline Slope method 1 first region & $0.29 \pm 0.01$ & $0.038 \pm 0.001$ & $0.47 \pm 0.05$ & $0.054 \pm 0.004$ \\
\hline Slope method 1 second region & - & $0.064 \pm 0.001$ & - & $0.092 \pm 0.01$ \\
\hline Contact method 2 first region & $\begin{array}{c}0.31 @ \\
u=14.6 \mathrm{~nm}\end{array}$ & $\begin{array}{c}0.037 @ \\
u=2.6 \mathrm{~nm}\end{array}$ & $\begin{array}{c}0.21 @ \\
u=2.7 \mathrm{~nm}\end{array}$ & $\begin{array}{c}-0.14 @ \\
u=3.4 \mathrm{~nm}\end{array}$ \\
\hline Contact method 2 second region & & $\begin{array}{c}0.045 @ u= \\
5.5 \mathrm{~nm}\end{array}$ & & $\begin{array}{c}0.21 @ \\
u=6.7 \mathrm{~nm}\end{array}$ \\
\hline Stick to first CM slip amplitude & $12.5 \mathrm{~nm}$ & $2.6 \mathrm{~nm}$ & $6 \mathrm{~nm}$ & $3.4 \mathrm{~nm}$ \\
\hline
\end{tabular}

condition ignores viscous dissipation while calculating $\mu$, which by the fits shown in Figure 5, is an appropriate assumption to make for air and DI water. However, the fit is poor in the case of $\mathrm{TiO}_{2}$, suggesting that this assumption may no longer be applicable. It is possible that the presence of NP lowers the dissipation at low oscillation amplitudes, replacing the contact with a more complex granular interface. Once this condition is relaxed, however, the values still do not show the expected reductions to friction, indicating that there are still difficulties with applying the model.

One possible explanation for the continued analysis failure is that the NPs possibly change the mechanics at the contacts, which may violate some of the underlying assumptions. One possibility of NPs affecting assumptions is that the CM slip regime as defined may no longer be present if rolling and/or slipping NP are present at the interface. Additionally, the amplitude of vibration can no longer be assumed to be much smaller than the size of the contact, since the contact may be comprised of $40 \mathrm{~nm}$ sized objects. Other model assumptions that could become problematic by the addition of NPs include the sphere being in contact with a ridged, flat surface, ignoring surface roughness, and assuming the contact area and slip annulus are uniform circles (Hanke et al., 2013). These assumptions could also be exacerbated by the potential formation of NP film formation at the contact points and/or the embedding of NPs into the surfaces. A visible residue was observed on the QCM after the $\mathrm{TiO}_{2}$ trial which remained even after rinsing the QCM with acetone and DI water, but we were unable to confirm the presence of embedded NP in the film by imaging. Complications to the contact introduced by the NPs could have a notable effect on the model because $\Delta \Gamma$ is equal to the area under the force-displacements loop divided by $\mathrm{u}_{\mathrm{o}}$, where the force displacement loop will have different shapesdepending on what slip regime the contact is in Hanke et al. (2013).

The sensitivity of $\Delta \Gamma$ tothe contact shape and conditions could also explain the behavior observed in $\Delta \Gamma$ in the stick regime. The small variations in $\Delta \Gamma$ at small amplitudes in air are likely due to small shifts and departures from the ideal model of perfect stick. This does not readily explain the largedepartures from expectation seen in the liquid trials at small amplitudes. The addition of liquids into the system resulted in low amplitude $\Delta \Gamma$ being largely negative with the shape either mostly concave down (DI) or concave up $\left(\mathrm{TiO}_{2}\right)$. Since this same behavior was not seen in the air experiments, it is likely the behavior is caused by the liquids. It is possible that at low amplitude, the observed $\Delta \Gamma$ behavior may be dominated by any energy dissipation in the liquid or behavior such as capillary action which might change the liquid barrier between contacts.

From another point of view, one might argue that the continuum model is highly successful even for the case of the $\mathrm{TiO}_{2}$ suspension. Given that it is only applicable in CM partial slip regimes, and the lack of a clear CM slip trend in the $\Delta \mathrm{f}$ and $\Delta \Gamma$ vs. $u$ data may indicate that there simply is no $\mathrm{CM}$ slip regime present in this complex interface, the model still manages to produce a value for $\mathrm{TiO}_{2}$ close to water. Since the CM slip regime will potentially result in the formation of wear particles (Fouvry et al., 1998), and these wear particles do not appear to have a negative impact on the model as seen in the air and water cases, then it is possible that the wear particles formed in the CM regime are less numerous than the NPs or that the wear particles were small enough to fall in the roughness gaps while the $40 \mathrm{~nm}$ NPs were not. Therefore, a follow-up study with varying sized NP would be of interest to determine the size of the particulate at which, if any, the model might become more applicable. In particular, if a collection of poorly coupled collection of sliding or rolling particles were pushed into the gaps, then the continuum behavior might be recovered. In contrast, If the smaller particles formed the contract itself, then the model assumption of an amplitude of vibration being far smaller than the NP contact size would be even less applicable.

\section{SUMMARY AND CONCLUSIONS}

Using a continuum analysis model, the response of a QCM to tripod 3[4SS/304SS rubbing contacts in air, water and a $\mathrm{TiO}_{2}$ suspension has been employed to evaluate friction coefficients. The results for dry and water lubricated contacts compare favorably with friction coefficient measurements reported in the literature for the same materials' combinations. The model appears less applicable to the case of contacts lubricates with the NP suspension. The continuum nature of the model does not however appear to be the dominant factor underlying its 
failure to apply to NP lubricated suspensions. The failure is likely attributed to a lack of a CM slip regime when NP are present at the interface, e.g., in the form of a poorly coupled collection of sliding or rolling particles, and/or the fact that the amplitude of vibration is no longer far smaller than the NP contact size regions.

\section{DATA AVAILABILITY STATEMENT}

All datasets generated for this study are included in the article/supplementary material.

\section{REFERENCES}

Acharya, B., Pardue, T. N., Avva, K. S., and Krim, J. (2018). In situ, real time studies of thermal reaction film formation temperatures for iron and 304ss surfaces immersed in 5\% tricresyl phosphate in base oil. Tribol. Int. 126, 106-115. doi: 10.1016/j.triboint.2018.04.034

Acharya, B., Pardue, T. N., Su, L., Smirnov, A. I., Brenner, D. W., and Krim, J. (2019a). Nanotribological performance factors for aqueous suspensions of oxide nanoparticles and their relation to macroscale lubricity. Lubricants 7:49. doi: 10.3390/lubricants7060049

Acharya, B., Seed, C. M., Brenner, D. W., Smirnov, A. I., and Krim, J. (2019b). Tuning friction and slip at solid-nanoparticle suspension interfaces by electric fields. Scient. Rep. 9:18584. doi: 10.1038/s41598-019-54515-1

Ali, M. K., Xianjun, H., Mai, L., Qingping, C., Turkson, R. F., and Bicheng, C. (2016). Improving the tribological characteristics of piston ring assembly in automotive engines using $\mathrm{Al}_{2} \mathrm{O}_{3}$ and $\mathrm{TiO}_{2}$ nanomaterials as nano-lubricant additives. Tribol. Int. 103, 540-554. doi: 10.1016/j.triboint.2016.08.011

Berg, S., and Johannsmann, D. (2003). High speed microtribology with quartz crystal resonators. Phys. Rev. Lett. 91:145505. doi: 10.1103/PhysRevLett.91.145505

Berg, S., Prellberg, T., and Johannsmann, D. (2003). Nonlinear contact mechanics based on ring-down experiments with quartz crystal resonators. Rev. Sci. Instrum. 74, 118-126. doi: 10.1063/1.1523647

Borovsky, B., Booth, A., and Manlove, E. (2007). Observation of microslip dynamics at high-speed microcontacts. Appl. Phys. Lett. 91:114101. doi: $10.1063 / 1.2784172$

Borovsky, B., Krim, J., Syed Asif, A., and Wahl, K. J. (2001). Measuring nanomechanical properties of a dynamic contact using an indenter probe and quartz crystal microbalance. J. App. Phys. 90:639. doi: 10.1063/1.1413493

Borovsky, B. P., Bouxsein, C., O’Neill, C., and Sletten, L. R. (2017). An integrated force probe and quartz crystal microbalance for high-speed microtribology. Tribol. Lett. 65:148. doi: 10.1007/s11249-017-0933-6

Borovsky, B. P., Garabedian, N. T., McAndrews, G. R., Wieser, R. J., and Burris, D. L. (2019). Integrates QCM-microtribometry: friction of single-crystal $\mathrm{MoS}_{2}$ and gold from $\mu \mathrm{m} / \mathrm{s}$ to m/s. ACS Appl. Mater. Interfaces. 11, 40961-40969. doi: 10.1021 /acsami.9b15764

Braiman, Y., Barhen, J., and Protopopescu, V. (2003). Control of friction at the nanoscale. Phys. Rev. Lett. 90:094301. doi: 10.1103/PhysRevLett.90.094301

Brizmer, V., Kligerman, Y., and Etsion, I. (2007). A model for junction growth of a spherical contact under full stick condition. J. Tribol. 129, 783-790. doi: $10.1115 / 1.2772322$

Ciavarella, M. (2015). Transition from stick to slip in Hertzian contact with "Griffith" friction: The Cattaneo-Mindlin problem revisited. J. Mech. Phys. Solids. 84, 313-324. doi: 10.1016/j.jmps.2015.08.002

Curtis, C. K., Marek, A., Smirnov, A. I., and Krim, J. (2017). A comparative study of the nanoscale and macroscale tribological attributes of alumina and stainless steel surfaces immersed in aqueous suspensions of positively or negatively charged nanodiamonds. Beil. J. Nanotechnolgy. 8, 2045-2059. doi: 10.3762/bjnano.8.205

\section{AUTHOR CONTRIBUTIONS}

$\mathrm{CS}, \mathrm{BA}$, and $\mathrm{JK}$ conceived the design of the experiment and constructed the apparatus. CS performed the experimental measurements and data analysis. CS and JK composed and edited the manuscript. All authors contributed to the article and approved the submitted version.

\section{FUNDING}

This work was supported by the US National Science Foundation, Materials Genome Initiative Project \#DMR1535082.

Dawson, B. D., Lee, S. M., and Krim, J. (2009). Tribo-induced melting transition at a sliding asperity contact. Phys. Rev. Lett. 103:205502. doi: 10.1103/PhysRevLett.103.205502

Dirri, F., Palomba, E., Longobardo, A., Zampetti, E., Saggin, B., and Scaccabarozzi, D. (2019). A review of quartz crystal microbalances for space applications. Sens. Actu. A Phys. 287, 48-75. doi: 10.1016/j.sna.2018.12.035

Dultsev, F. N., and Nekrasov, D. V. (2018). Treatment of the resonance curve recorded during measurement of the signal of particle rupture from the QCM surface. Sens. Actu. B Chem. 15, 70-75. doi: 10.1016/j.snb.2018.04.029

Ellis, J. S., McHale, G., Hayward, G. L., and Thompson, M. (2003). Contact anglebased predictive model for slip at the solid-liquid interface of a transverse-shear mode acoustic wave device. J. App. Phys. 94, 6201-6207. doi: 10.1063/1.1619195

Etsion, I. (2010). Revisiting the cattaneo-mindlin concept of the interfacial slip in tangentially loaded compliant bodies. J. Tribol. 132:20801. doi: 10.1115/1.4001238

Fouvry, S., Kapsa, P., and Vincent, L. (1998). Developments of fretting sliding criteria to quantify the local friction coefficient evolution under partial slip condition. Tribol. Series. 34, 161-172. doi: 10.1016/S0167-8922(98)80071-0

Glavatskih, S., and Höglund, E. (2008). Tribotronics-towards active tribology. Tribol. Int. 41, 934-939. doi: 10.1016/j.triboint.2007.03.001

Han, T., Nag, A., Mukhopadhyay, S. C., and Xu, Y. (2019). Carbon nanotubes and its gas-sensing applications: a review. Sens. Actu. A Phys. 291, 107-143. doi: 10.1016/j.sna.2019.03.053

Hanke, S., Petri, J., and Johannsmann, D. (2013). Partial slip in mesoscale contacts: dependence on contact size. Phys. Rev. E. 88:032408. doi: 10.1103/PhysRevE.88.032408

Hsu, S., Ying, C., and Zhao, F. (2014). The nature of friction: a critical assessment. Friction 2, 1-26. doi: 10.1007/s40544-013-0033-z

Johannsmann, D. (2007). Studies of contact mechanics with the QCM. Springer Ser Chem Sens Biosens. 5, 151-170. doi: 10.1007/5346-026

Johannsmann, D., Reviakine, I., and Richter, R. (2009). Dissipations in films of adsorbed nanospheres studied by quartz crystal microbalance (QCM). Anal. Chem. 81, 8167-8176. doi: 10.1021/ac901381z

Josse, F., and Lee, Y. (1998). Analysis of the radial dependence of the mass sensitivity for modified-electrode quartz crystal resonators. Anal. Chem. 70, 237-247. doi: 10.1021/ac9706032

Kim, H. J., and Kim, D. E. L. (2009). Nano-scale friction: a review. Precis. Eng. Manuf. 10, 141-151. doi: 10.1007/s12541-009-0039-7

Krim, J. (2012). Friction and energy dissipation mechanisms in adsorbed molecules and molecularly thin films. Adv. Phys. 61:3, 155-323. doi: 10.1080/00018732.2012.706401

Krim, J. (2019). Controlling friction with external electric or magnetic fields: 25 examples. Front. Mech. Eng. 5:22. doi: 10.3389/fmech.2019.00022

Laschitsch, A., and Johannsmann, D. (1999). High frequency tribological investigations on quartz resonator surfaces. J. Appl. Phys. 85:3759. doi: $10.1063 / 1.369745$

Leopoldes, J., and Jia, X. (2010). Transverse shear oscillator investigation of boundary lubrication in weakly adhered films. Phys. Rev. Lett. 105:266101. doi: 10.1103/PhysRevLett.105.266101 
Lu, F., Lee, H. P., and Lim, S. P. (2004). Quartz crystal microbalance with rigid mass partially attached on electrode surfaces. Sens. Actu. A Phys. 112, 203-210. doi: 10.1016/j.sna.2004.01.018

Lu, X., Khonsari, M. M., and Gelinck, E. R. (2006). The Stribeck curve: experimental results and theoretical prediction. J. Tribol. 128, 789-794. doi: $10.1115 / 1.2345406$

Martin, B. A., and Hager, H. E. (1989). Velocity profile on quartz crystals oscillating in liquids. J. Appl. Phys. 65:2630. doi: 10.1063/1.342772

M'boungui, G., Semail, B., Giraud, F., and Jimoh, A. A. (2014). Development of a novel plane piezoelectric actuator using Hamilton's principle based model and Hertz contact theory. Sens. Actu. A Phys. 217, 116-123. doi: $10.1016 /$ j.sna.2014.06.026

Paggi, M., Pohrt, R., and Popov, V. L. (2014). Partial-slip frictional response of rough surfaces. Sci Rep. 4:5178. doi: 10.1038/srep05178

Pardue, T. N., Acharya, B., Curtis, C. K., and Krim, J. (2018). A tribological study of $\Gamma-\mathrm{Fe}_{2} \mathrm{O}_{3}$ nanoparticles in aqueous suspension. Tribol. Lett. 66:130. doi: 10.1007/s11249-018-1083-1

Popov, V. L. (2010). "Chapter 2: qualitative treatment of contact problemsnormal contact without adhesion," in Contact Mechanics and Friction: Physical Principles and Applications (Berlin; Heidelberg: Springer Verlag), 9-25. doi: 10.1007/978-3-642-10803-7_3

Rodahl, M., and Kasemo, B. (1996). On the measurement of thin liquid overlayers with the quartz-crystal microbalance. Sens. Actu. A Phys. 54, 448-456. doi: 10.1016/S0924-4247(97)80002-7

Seed, C. M., Acharya, B., Andrus, R., and Krim, J. (2020). Correlation of high frequency QCM sphere-plate stiffness measurements with macroscopic frictional contacts in thin film and bulk stainless steel materials. Sens. Actu. A Phys. 36:111913. doi: 10.1016/j.sna.2020.111913

Sen, S., Chow, C. M., Moazzen, E., Segre, C. U., and Timofeeva, E. V. (2017). Electroactive nanofluids with high solid loading and low viscosity for rechargeable redox flow batteries. J. Appl. Electrochem. 47, 593-605. doi: 10.1007/s10800-017-1063-4

Vittorias, E., Kappl, M., Butt, H. J., and Johannsmann, D. (2010). Studying mechanical microcontacts of fine particles with the quartz crystal microbalance. Powder Technol. 203, 489-502. doi: 10.1016/j.powtec.2010. 06.011
Vlachová, J., König, R., and Johannsmann, D. (2015). Stiffness of sphereplate contacts at $\mathrm{MHz}$ frequencies: dependence on normal load, oscillation amplitude, and ambient medium. Beils. J. Nanotechnol. 6, 845-856. doi: 10.3762/bjnano.6.87

Wang, J., Polleux, J., Lim, J., and Dunn, B. (2007). Pseudocapacitive contributions to electrochemical energy storage in $\mathrm{TiO}_{2}$ (Anatase) nanoparticles. J. Phys. Chem. C. 111, 14925-14931. doi: 10.1021/jp074464w

Wang, L., Junkuo, G., and Xu, J. (2019). QCM formaldehyde sensing materials: design and sensing mechanism. Sens. Actu. B Chem. 293, 71-82. doi: 10.1016/j.snb.2019.04.050

Wu, H., Jia, F., Li, Z., Lin, F., Huo, M., Huang,. S., et al. (2020). Novel water-based nanolubricant with superior tribological performance in hot steel rolling. Int. J. Extr. Manuf. 2:025002. doi: 10.1088/2631-7990/ab82fe

Yamaguchi, T., and Hokkirigawa, K. (2016). Friction and wear properties of PEEK resin filled with $\mathrm{RB}$ ceramics particles under water lubricated condition. Tribol. Online. 11, 653-660. doi: 10.2474/troll.11.653

Yu, X., Zhou, J., and Jiang, Z. (2016). Developments and possibilities for nanoparticles in water-based lubrication during metal processing. Rev. Nanosci. Nanotechnol. 5,136-163. doi: 10.1166/rnn.2016.1072

Zhang, C., and Wang, Z. L. (2016). Tribotronics-A new field by coupling triboelectricity and semiconductor. Nano Today. 11, 521-536. doi: 10.1016/j.nantod.2016.07.004

Zhang, X., and Li, Q. (2010). Enhancement of friction between carbon nanotubes: an efficient strategy to strengthen fibers. ACS Nano. 4, 292-316. doi: $10.1021 / \mathrm{nn} 901515 \mathrm{j}$

Conflict of Interest: The authors declare that the research was conducted in the absence of any commercial or financial relationships that could be construed as a potential conflict of interest.

Copyright $(2020$ Seed, Acharya and Krim. This is an open-access article distributed under the terms of the Creative Commons Attribution License (CC BY). The use, distribution or reproduction in other forums is permitted, provided the original author(s) and the copyright owner(s) are credited and that the original publication in this journal is cited, in accordance with accepted academic practice. No use, distribution or reproduction is permitted which does not comply with these terms. 